\title{
Los géneros Alysicarpus y Desmodium (Fabaceae) en la península de Yucatán, México
}

\section{The genus Alysicarpus and Desmodium (Fabaceae) in theYucatán Peninsula, Mexico}

\author{
Leticia Torres-Colín ${ }^{1 凶}$, Rodrigo Duno-de Stefano ${ }^{2}$ y Carlos Gómez-Hinostrosa ${ }^{1}$
}

${ }^{1}$ Departamento de Botánica, Instituto de Biología, Universidad Nacional Autónoma de México. Apartado postal 70-367, 04510 México, D. F., México. ${ }^{2}$ Herbario CICY, Centro de Investigación Científica de Yucatán, A. C. Calle 43 Núm. 30, Col. Chuburná de Hidalgo, 97200 Mérida, Yucatán, México. \lety@ibiologia.unam.mx

\begin{abstract}
Resumen. Se presenta una revisión de los géneros Alysicarpus Desv. y Desmodium Desv. (Desmodieae: Desmodiinae) en la porción mexicana de la península de Yucatán. Basada en ejemplares de herbario depositados en 8 herbarios CHAPA, CICY, CIQRO, ENCB, IEB, MEXU, UCAM y XAL. Se reconocen Alysicarpus vaginalis (L.) DC., Desmodium affine Schltdl., D. distortum (Aubl.) J.F. Macbr., D. glabrum (Mill.) DC., D. incanum DC., D. procumbens (Mill.) Hitchc., D. scorpiurus (Sw.) Desv., D. tortuosum (Sw.) DC. y D. triflorum (L.) DC. Se incluyen claves para la identificación de géneros y especies, así como descripciones morfológicas, información ecológica, fenológica, nombres comunes (cuando se conocen), algunos usos y mapas de distribución.
\end{abstract}

Palabras clave: Alysicarpus, Desmodium, Fabaceae, Campeche, Quintana Roo, Yucatán, México.

\begin{abstract}
A revision of the genera Alysicarpus Desv. and Desmodium Desv. (Desmodieae: Desmodiinae) for the Mexican region of the Yucatán Peninsula is presented. This treatment is based on the study of botanical material of 8 herbaria, CHAPA, CICY, CIQRO, ENCB, IEB, MEXU, UCAM, and XAL. We recognized Alysicarpus vaginalis (L.) DC., Desmodium affine Schltdl., D. distortum (Aubl.) J.F. Macbr., D. glabrum (Mill.) DC., D. incanum DC., D. procumbens (Mill.) Hitchc., D. scorpiurus (Sw.) Desv., D. tortuosum (Sw.) DC., and D. triflorum (L.) DC. Identification keys to genera and to species are included, as well as morphological descriptions, ecology, phenology, some common names and uses for each species.
\end{abstract}

Key words: Alysicarpus, Desmodium, Fabaceae, Campeche, Quintana Roo, Yucatán, Mexico.

\section{Introducción}

La región mexicana de la península de Yucatán (PY) está conformada políticamente por los estados de Campeche, Quintana Roo y Yucatán al sureste de México, situada entre los paralelos $17^{\circ} 49^{\prime}-21^{\circ} 37^{\prime} \mathrm{N}$ y los meridianos $86^{\circ} 42^{\prime}-92^{\circ} 28^{\prime} \mathrm{O}$ (INEGI, 2000). Aunque existen algunas clasificaciones biogeográficas para la región sur y sureste del país (Barrera, 1962; Rzedowski, 1978; Morrone, 2005) por razones prácticas para este trabajo y como parte de la revisión del género Desmodium por la primera autora, restringimos nuestra área de estudio sólo a los 3 estados de la península anteriormente mencionados.

Los géneros Alysicarpus y Desmodium son los únicos representantes de la tribu Desmodieae (Benth.) Hutch. para la PY. Alysicarpus contiene unas 30 especies distribuidas en los trópicos del Viejo Mundo (Ohashi,

Recibido: 17 marzo 2010; aceptado: 14 marzo 2011
2005), de las cuales sólo A. vaginalis (L.) DC., se ha introducido a los trópicos de América y se ha encontrado recientemente en algunas áreas de la península.

Desmodium es un género con 275 especies distribuidas en los trópicos y subtrópicos de todo el mundo (Ohashi, 2005) y con una gran diversidad en México (cerca de 100 especies, Torres-Colín en preparación). A la fecha no existe un trabajo que incluya todas las especies mexicanas, por lo que la fuente más importante de información para el género han sido los estudios florísticos y algunas floras regionales (Hemsley, 1887; Standley, 1930; 1946; Cowan, 1983; Sousa y Cabrera, 1983; Breedlove, 1986; McVaugh, 1987; Ibarra y Sinaca, 1987; Téllez y Cabrera, 1987; Flores, 1992; Torres et al., 1997; Duran et al., 2000; Torres-Colín, 2005; Sosa et al, 1985; Torres-Colín y Delgado-Salinas, 2008).

Algunos de estos estudios incluyen especies de Desmodium presentes en la PY, y el número difiere de un autor a otro y hasta ahora se han reconocido a D. adscendens (Sw.) DC., D. distortum (Aubl.) J. F. Macbr., D. frutescens Schindl., D. glabrum (Mill.) DC., 
Clave para los géneros Alysicarpus y Desmodium

1. Hojas 1-folioladas, estípulas generalmente connadas; cáliz profundamente 5-dividido, glumáceo, estriado; flores generalmente 2 por nudo; lomentos subcilíndricos, comprimidos; artículos simétricos en el margen, se tornan negros al madurar. Alysicarpus 1. Hojas generalmente 3-folioladas, estípulas libres o amplexicaules; cáliz 5-dividido, aparentando 2 labios subiguales, no estriados; flores 2-6 por nudo; lomentos aplanados o espiralados; artículos asimétricos en el margen, nunca se tornan negros al madurar.

Desmodium

D. incanum DC., D. molle (Vahl) DC., D. procumbens (Mill.) Hitchc., D. procumbens (Mill.) Hitchc. var. longipes (Schindl) B. G. Schub., D. procumbens (Mill.) Hitche var. procumbens, D. procumbens (Mill.) Hitchc. var. typicum, $D$. purpureum (Mill.) Fawc. et Rendle, $D$. scorpiurus (Sw.) Desv. y D. tortuosum (Sw.) DC.

Considerando lo anterior y como parte de los estudios que se vienen realizando para la PY, decidimos revisar las especies de los géneros Alysicarpus y Desmodium, así como favorecer su reconocimiento con claves de identificación y descripciones morfológicas.

\section{Materiales y métodos}

Se revisaron 294 ejemplares de los herbarios CHAPA, CICY, CIQRO, ENCB, IEB, MEXU, UCAM y XAL. A partir de ellos se elaboraron descripciones morfológicas, citando sinonimia, se proporcionan claves para géneros y especies, algunos datos de hábitat, nombres comunes y usos, además de representar su distribución en mapas generados con el programa ArcView versión 3.2 y editados en Adobe Ilustrator.

Se registran 2 géneros: Alysicarpus con 1 especie y Desmodium con 8 especies.

\section{Descripciones}

\section{Alysicarpus Desv.} Alysicarpus Desv., J. Bot. Agric.1: 120. 1813.

Hierbas anuales o perennes, suberectas o procumbentes. Tallos estriados, pubescencia con tricomas uncinados o glabrescentes. Hojas alternas, 1-folioladas (rara vez 3-folioladas); estípulas persistentes, acuminadas, libres o connadas, erectas, glabrescentes; pecíolos sulcados adaxialmente; estipelas 2 en el folíolo terminal y una en cada lateral; folíolos linear-lanceolados, elípticos a ovados. Inflorescencias terminales, rara vez axilares, en pseudoracimos; brácteas primarias deciduas, estriadas, glabrescentes; bractéolas ausentes. Flores rosa o púrpura; cáliz profundamente 5-dividido, glumáceo, estriado, lóbulos desiguales, el superior connado, entero o ligeramente 2-dividido, lóbulo inferior con dientes libres o imbricados; corola con estandarte obovado a orbicular, unguiculado, con 2 pequeños pliegues en la base; alas oblicuamente oblongas, pétalos de la quilla conniventes; androceo diadelfo, estambre vexilar libre; gineceo con ovario sésil o cortamente estipitado, óvulos numerosos; estilo incurvado en el ápice, estigma generalmente capitado. Lomentos sésiles, subcilíndricos, indehiscentes, artículos 2-8, oblongos, margen simétrico, reticulados, puberulentos a glabrescentes; semillas 1 por artículo, suborbiculares a globosas.

Alysicarpus vaginalis (L.) DC., Prodr. 2:353. 1825. Hedysarum vaginale L., Sp. Pl. 2:746. 1753. Tipo: Sri Lanka (Ceilán), Herb. Hermann 1:27 No. 287 (lectotipo: BM, designado por Verdcourt, 1997).

Hierbas perennes, suberectas o postradas, $35-40 \mathrm{~cm}$ altura. Tallos estriados, escasamente uncinados a glabrescentes. Hojas 1-folioladas; estípulas persistentes, connadas, 1.5$2.0 \mathrm{~mm}$ largo, 0.3-0.8 $\mathrm{mm}$ ancho, lanceoladas, glabras en ambas superficies; pecíolos estriados, $0.5-2.0 \mathrm{~cm}$ largo, glabros; estipelas $0.5-0.7 \mathrm{~mm}$ largo, ca. $0.2 \mathrm{~mm}$ ancho, subuladas, glabras en ambas superficies; peciólulos 1.0 $\mathrm{mm}$ largo, glabros; folíolos $0.5-5.5 \mathrm{~cm}$ largo, $0.4-0.7 \mathrm{~cm}$ ancho, oblongos, base subcordada, ápice agudo, mucronado, haz glabrescente, envés uncinado y con la vena media fuertemente impresa y estrigosa. Inflorescencias terminales, $5.0-10.0 \mathrm{~cm}$ de largo; eje de la inflorescencia glabro; brácteas primarias deciduas, 5.0-6.3 $\mathrm{mm}$ largo, ca. $1.3 \mathrm{~mm}$ ancho, subuladas, ciliadas; brácteas secundarias deciduas, 2.2-2.5 mm largo, 1.0-1.3 $\mathrm{mm}$ ancho, adaxialmente uncinadas, abaxialmente glabras; pedicelos ca. 1.5 $\mathrm{mm}$ largo, uncinados. Flores púrpura, 2 por nudo; cáliz ca. $4.3 \mathrm{~mm}$ largo, puberulento en ambas superficies, tubo 1.8-2.0 mm largo, dientes 1.8- $2.6 \mathrm{~mm}$ largo, acuminados, ciliados; corola 5.0-6.0 mm largo, estandarte 5.0-5.5 mm largo, $3.0 \mathrm{~mm}$ ancho, obovado, alas $5.5 \mathrm{~mm}$ largo, 1.5 $\mathrm{mm}$ ancho, oblicuamente oblongas, quilla $5.8 \mathrm{~mm}$ largo, con los pétalos conniventes, $5.8 \mathrm{~mm}$ largo, ovario sésil, puberulento, 7 óvulos. Lomentos sésiles, subcilíndricos, comprimidos; artículos 6-7, 2.5-3.0 mm largo, 1.5-2.0 mm ancho, oblongos, margen entero, uncinados, indehiscentes, negros cuando maduros; semillas $1.5 \mathrm{~mm}$ largo, $1.3 \mathrm{~mm}$ ancho, suborbiculares.

Distribución y hábitat. Alysicarpus vaginalis es una especie nativa del Viejo Mundo e introducida a los trópicos 
de América. En México se localiza en los estados de Chiapas, Tabasco y Veracruz, en vegetación secundaria. Por el momento no podemos concluir que esta especie sea escapada y naturalizada en la región, ya que en los últimos 3 años se ha localizado en los márgenes de varios campos deportivos de la ciudad de Mérida y alrededores, donde se utilizan pastos importados como cubiertas de terreno (Fig. 1).

Comentarios taxonómicos. Esta especie representa una novedad para la zona y sólo se ha mencionado para Belice y Guatemala, áreas geográficas no consideradas como parte de la PY en este trabajo (Standley et Steyermark, 1946). Es fácilmente reconocible por sus hojas 1-folioladas, flores con el cáliz glumáceo y frutos subcilíndricos, comprimidos, de color negro.

Material examinado. Yucatán: R. Duno 2045 (CICY).

Desmodium Desv.

Desmodium Desv., J. Bot. Agric. 1: 122. 1813.

Meibomia Heist ex Fabr., Enum. 168. 1759.

Hierbas anuales o perennes, erectas, postradas o procumbentes. Tallos estriados o sulcados, con tricomas simples, uncinados y glandulosos o glabrescentes. Hojas alternas generalmente 3- folioladas (ocasionalmente 1-5 folioladas); estípulas persistentes o deciduas, libres o amplexicaules, erectas o retrorsas, pubescentes o glabras; pecíolos estriados o sulcados adaxialmente, raquis generalmente corto, puberulento; estipelas 2 en el folíolo terminal y 1 en cada lateral; folíolos linear-lanceolados, ovados, elípticos, oblongos. Inflorescencias axilares y terminales, en pseudoracimos, panículas o fascículos; brácteas primarias persistentes o deciduas, estriadas, ciliadas, sosteniendo generalmente 2 o más flores; brácteas secundarias persistentes o deciduas, estriadas, ciliadas, sosteniendo 1 flor. Flores rosa, lila, púrpura u ocasionalmente blancas; cáliz 2-labiado, lóbulo superior 2-dividido, el inferior 3-dentado, 2 dientes laterales y 1 diente central; corola con estandarte ovado, obovado u oblongo, en ocasiones maculado, ligeramente unguiculado, alas generalmente oblongas, unguiculadas, unidas a la quilla en la base; quilla con pétalos parcialmente fusionados en la porción distal, unguiculados; androceo diadelfo, estambre vexilar libre o parcialmente fusionado en la base; gineceo con ovario sésil o estipitado, puberulento o glabro, óvulos 2 o más; estilo delgado, glabro; estigma terminal. Lomentos sésiles o estipitados, aplanados o espiralados, indehiscentes; artículos 1-8, orbiculares, oblongos, rómbicos, cuadrados, planos; revoluto-involuto en el margen, reticulados, uncinado-puberulentos a glabrescentes, ocasionalmente ciliados; semillas 1 por artículo, orbiculares, oblongas u rómbicas.

Clave para identificar las especies de Desmodium

1. Inflorescencias axilares en fascículos; flores 2-4 por nudo; hierbas postradas; folíolos obovados, de ca. $1.0 \mathrm{~cm}$ de largo. D. triflorum

1. Inflorescencias axilares y terminales en pseudoracimos o panículas; flores 2-6 por nudo; hierbas erectas o procumbentes en ocasiones sufruticosas; folíolos ovados, angostamente ovados o elípticos, de más de $1.0 \mathrm{~cm}$ largo.

2. Lomentos sésiles, un sólo artículo fértil D. glabrum

2. Lomentos sésiles o cortamente estipitados, todos los artículos fértiles.

3. Hierbas anuales, 2-5 flores por nudo. D. procumbens

3. Hierbas generalmente perennes, 2 flores por nudo.

4. Lomentos sésiles con artículos oblongos, margen ligeramente sinuado D. scorpiurus

4. Lomentos sésiles o estipitados con artículos elípticos u oblongos, margen desigual, el superior entero, ligeramente sinuado o crenado y el inferior crenado.

5. Estípulas amplexicaules, ovadas, ca. $1.5 \mathrm{~cm}$ de largo.

6. Pedicelos de $1.0 \mathrm{~cm}$ de largo; lomentos estipitados; artículos generalmente glabros........D. distortum 6. Pedicelos de ca. $2.0 \mathrm{~cm}$ de largo; lomentos sésiles; artículos puberulentos, mezclados con tricomas uncinados. D. tortuosum

5. Estípulas libres o unidas en el lado opuesto del tallo, lanceoladas, ovadas u ovado-lanceoladas, hasta $1.0 \mathrm{~cm}$ de largo.

7. Flores blancas, pedicelos de $2.0 \mathrm{~cm}$ de largo; lomentos con 4-6 artículos. ..D. affine

7. Flores lila, pedicelos de menos de $1.0 \mathrm{~cm}$ de largo; lomentos con 6-8 artículos ..D. incanum 


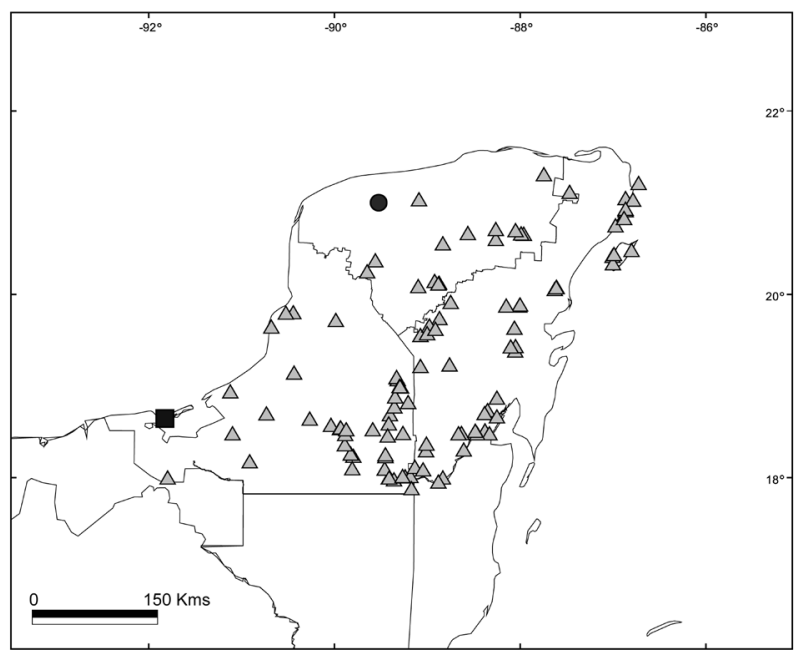

Figura 1. Distribución de Alysicarpus vaginalis (•); Desmodium incanum ( $\Delta$ ) y D. triflorum (ם).

Desmodium affine Schltdl., Linnaea 12:312.1838. Meibomia affinis (Schltdl.) Kuntze, Revis. Gen. Pl. 1: 197. 1891. Tipo: México.Veracruz: prope Hacienda de La Laguna, Schiede s.n., aug 1829 (lectotipo: HAL).

Desmodium albiflorum Salzm. ex Benth., Fl. Bras. 15:99. 1859. Meibomia albiflora (Salzm. ex Benth.) Kuntze, Revis. Gen. Pl. 1: 197. 1891. Tipo: Brasil. Bahia, prope Bahiam, Salzmann s.n., s.f. (isotipos: GH, US!).

Hierbas perennes, procumbentes, $1.5 \mathrm{~m}$ de altura. Tallos con abundantes raíces adventicias, estriados, pilosos y ligeramente uncinados. Hojas 3-folioladas; estípulas persistentes, libres, $8.0-10.0 \mathrm{~mm}$ largo, $1.8-3.0 \mathrm{~mm}$ ancho, lanceoladas, subcordadas en la base, adaxialmente glabrescentes, abaxialmente glabras, ciliadas; pecíolos sulcados, 2.0-5.5 cm largo, pilosos y uncinados; estipelas $3.0-4.8 \mathrm{~mm}$ largo, ca. $0.4 \mathrm{~mm}$ ancho, subuladas, abaxialmente puberulentas y uncinadas, adaxialmente glabrescentes, ciliadas; peciólulos 1.5-2.0 mm largo, hirsutos; folíolo terminal 4.0$5.0 \mathrm{~cm}$ largo, $2.5-3.9 \mathrm{~cm}$ ancho, los laterales $3.0-4.0 \mathrm{~cm}$ largo, 1.5-3.0 cm ancho, ovados, base obtusa, ápice agudo, haz glabrescente, envés estrigoso y escasamente uncinado. Inflorescencias terminales, $10.0-18.0 \mathrm{~cm}$ largo; eje de la inflorescencia puberulento y uncinado; brácteas primarias deciduas, 2.0-3.0 mm largo, 0.5-1.0 $\mathrm{mm}$ ancho, subuladas, adaxialmente uncinadas, abaxialmente glabras, ciliadas; brácteas secundarias deciduas (no vistas); pedicelos 2.0 $\mathrm{cm}$ largo, uncinados. Flores blancas, 2 por nudo; cáliz $4.5 \mathrm{~mm}$ largo, adaxialmente hírtulo, abaxialmente glabro, lóbulo superior 2-dividido, $3.0 \mathrm{~mm}$ largo, lóbulo inferior con dientes laterales $2.8-3.0 \mathrm{~mm}$ largo, diente central 3.5 $\mathrm{mm}$ largo; corola ca. $7.0 \mathrm{~mm}$ largo, estandarte $6.0 \mathrm{~mm}$ largo, $5.0 \mathrm{~mm}$ ancho, obovado, alas $6.5 \mathrm{~mm}$ largo, $2.3 \mathrm{~mm}$ ancho, oblongas, quilla ca. $6.0 \mathrm{~mm}$ largo; ovario estipitado,

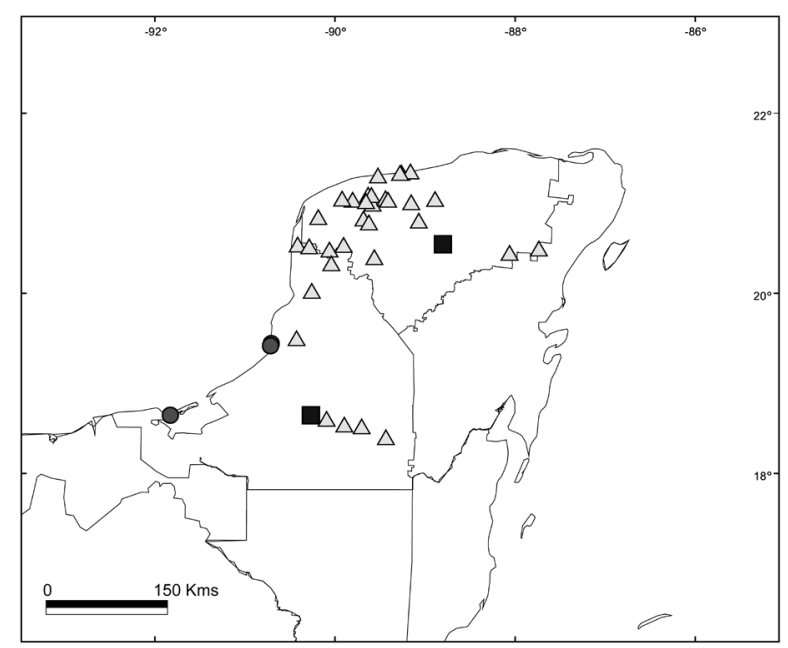

Figura 2. Distribución de Desmodium affine (匹); D. glabrum (А) y D. scorpiurus $(\bullet)$.

pubescente, 6 óvulos. Lomentos aplanados, corto estipitados; artículos 4-6, 6.0-7.0 mm largo, ca. $3.0 \mathrm{~mm}$ ancho, oblongos, margen superior ligeramente sinuoso, margen inferior crenado, uncinados, indehiscentes; semillas 3.5 $\mathrm{mm}$ largo, $2.0 \mathrm{~mm}$ ancho, elípticas.

Distribución y hábitat. Escasa en Campeche y Yucatán (Fig. 2). En otros estados de México (Chiapas, Colima, Guerrero, Jalisco, Michoacán, Morelos, Nayarit, Oaxaca, Puebla, San Luis Potosí, Sinaloa, Tabasco, Tamaulipas y Veracruz), Centroamérica, Antillas y Sudamérica. Habita en selva media subperennifolia y vegetación secundaria. En el poco material representado se observó floración de septiembre a abril y fructificación en octubre.

Comentarios taxonómicos. Históricamente, los ejemplares correspondientes a $D$. affine fueron determinados como $D$. incanum nombre que fue conservado por años; sin embargo, durante esta revisión encontramos algunos ejemplares de $D$. affine con caracteres típicos, como los pecíolos más largos que las hojas, los folíolos ovados, las flores blancas largamente pediceladas y los lomentos con 6 artículos. Aunque esta especie sólo se había mencionado para el estado de Campeche por Gutiérrez-Báez, (2003) representa una novedad florística no destacada por dicho autor.

Material examinado. Campeche: E. Ucan 1681 (CICY, ENCB, MEXU, XAL); Yucatán: A. Vara 360, 544 (CHAPA).

Desmodium distortum (Aubl.) J.F. Macbr., Publ. Field Columbian Mus., Bot. Ser. 8:101. 1930. Hedysarum distortum Aubl., Hist. Pl. Guiane 2:774. 1775. Meibomia distorta (Aubl.) Schindl., Repert. Spec. Nov. Regni Veg. 22:281. 1924. Tipo: Guayana Francesa, Aublet s.n., s.f. (probable lectotipo: BM). 
Desmodium hirsutum M. Martens et Galeotti, Bull. Acad. Roy. Sci. Bruxelles 10: 186. 1843. Meibomia hirsuta (M. Martens et Galeotti) Kuntze, Revis. Gen. Pl. 1:198. 1891. Tipo: México. Zacuapan et Mirador, Galeotti 3275, oct 1840 (isotipos: $F$ !, G, K!).

Hierbas perennes, erectas, $0.5-2.0 \mathrm{~m}$ de altura. Tallos estriados, puberulentos y uncinados. Hojas 3-folioladas; estípulas persistentes, amplexicaules, $6.5-15.0 \mathrm{~mm}$ largo, 4.0-6.5 mm ancho, ovadas, largamente acuminadas, glabras en ambas superficies, largamente ciliadas; pecíolos estriados, 2.0-11.0 cm largo, puberulentos y uncinados; estipelas $0.6-1.3 \mathrm{~cm}$ largo, $1.3 \mathrm{~mm}$ ancho, subuladas, puberulentas en ambas superficies, ciliadas; peciólulos 1.5-3.0 $\mathrm{mm}$ largo, hirsutos y escasamente uncinados; folíolo terminal $4.2-12.0 \mathrm{~cm}$ largo, $1.6-5.5 \mathrm{~cm}$ ancho, los laterales 3.0-7.0 cm largo, $1.6-3.5 \mathrm{~cm}$ ancho, ovados, ovado-lanceolados a elípticos, base obtusa, ápice agudo, haz glabrescente, envés escasamente uncinado en la vena media y secundarias. Inflorescencias axilares y terminales, en panículas, 10.0-37.0 cm largo; eje de la inflorescencia densamente uncinado, puberulentos y glanduloso; brácteas primarias deciduas, 3.0-6.0 mm largo, 0.5-0.6 mm ancho, lanceoladas, adaxialmente hírtulas, abaxialmente glabrescentes, ciliadas; brácteas secundarias deciduas, $2.0 \mathrm{~mm}$ largo, $0.2 \mathrm{~mm}$ ancho, subuladas, glabrescentes en ambas superficies, ciliadas; pedicelos $0.4-1.0 \mathrm{~cm}$ largo, puberulentos y uncinados. Flores de color lila, 2 por nudo; cáliz $3.8 \mathrm{~mm}$ largo, adaxialmente hirsútulo, abaxialmente glabro; lóbulo superior 2-dividido, $1.7 \mathrm{~mm}$ largo, lóbulo inferior con dientes laterales $1.7 \mathrm{~mm}$ largo, diente central $2.0 \mathrm{~mm}$ largo; corola $6.3 \mathrm{~mm}$ largo, estandarte $6.0 \mathrm{~mm}$ largo, $3.5 \mathrm{~mm}$ ancho, obovado, alas 5.5-6.0 $\mathrm{mm}$ largo, 2.0$3.0 \mathrm{~mm}$ ancho, oblongas, quilla $6.5-6.7 \mathrm{~mm}$ largo; ovario estipitado, glabro, 7 óvulos. Lomentos aplanados, estipitados; artículos 2-7, 3.5-4.5 cm largo, 2.5-4.0 mm ancho, elípticos, margen crenado, glabros, indehiscentes; semillas 2.2-2.4 mm largo, $1.5 \mathrm{~mm}$ ancho, oblongas.

Distribución y hábitat. Se distribuye en Campeche y escasamente en Quintana Roo y Yucatán (Fig. 3). En otros estados de México (Chiapas, Chihuahua, Colima, Guerrero, Jalisco, México, Michoacán, Morelos, Nayarit, Oaxaca, Puebla, Tabasco, Tamaulipas, Veracruz y Zacatecas), Centroamérica, Cuba y Sudamérica. Crece en selva baja caducifolia, selva media subperennifolia y vegetación secundaria. Florece y fructifica desde junio hasta marzo.

Comentarios taxonómicos. Desmodium distortum presenta afinidades con $D$. tortuosum y se les puede encontrar cohabitando en algunas regiones de la península. Se diferencia claramente de ésta última por sus flores que se desarrollan en pedicelos cortos de ca. $1.0 \mathrm{~cm}$ largo y por sus lomentos generalmente estipitados con artículos más pequeños y glabrescentes.

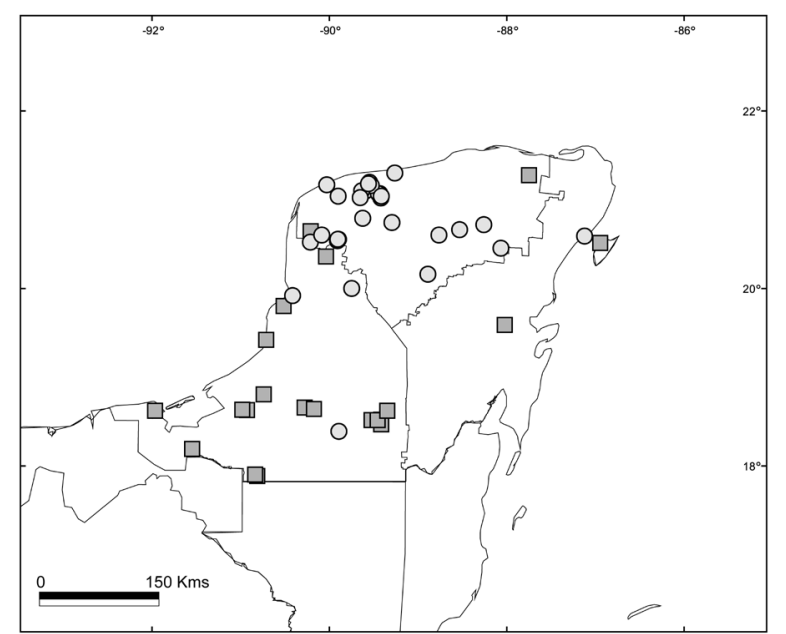

Figura 3. Distribución de Desmodium distortum (匹) y D. procumbens $(\bullet)$.

Material examinado. Campeche: C. Chan 4598, 4950 (CICY, MEXU); C. Gutiérrez 5116 (CICY), 5997 (CICY, MEXU); D. Álvarez 6546 (MEXU); E. Cabrera 3132 (MEXU), 10928 (CICY, CIQRO, MEXU, MO), 12619 (IEB, MEXU), 12737, 14397 (MEXU), 15979 (MEXU, IEB); G. Bacab 165 (MEXU); J.S. Flores 9036 (MEXU, XAL), 9985 (CICY, MEXU, XAL); J. Palmillas 746 (MEXU); M. Sousa 12028, 12238 (MEXU); O. Vela 676 (MEXU); Quintana Roo: E. Cabrera 10510 (MEXU); O. Téllez 2401 (CIQRO, MEXU), 3707 (ENCB, MEXU); Yucatán: C. Chan 7056 (CICY, MEXU); E. Cabrera 15712 (MEXU).

Desmodium glabrum (Mill.) DC., Prod. 2:338. 1825. Hedysarum glabrum Mill., Gard. Dict. ed. 8. Hedysarum no. 12. 1768. Meibomia glabra (Mill.) Kuntze, Revis. Gen. Pl. 1:198. 1891. Tipo: México. Campeche, Houstoun s.n., s.f. (holotipo: BM!). Hedysarum molle Vahl, Symb. Bot. 2:83. 1791. Desmodium molle (Vahl) DC., Prodr. 2: 332. 1825. Meibomia mollis (Vahl) Kuntze, Revis. Gen. Pl. 1:198. 1891. Tipo: Estados Unidos. Islas Vírgenes: ex insula St. Croix, Pflug s.n., 1820 (holotipo: C). Desmodium campestre Brandegee, Univ. Calif. Publ. Bot. 6:53. 1914. Tipo: México. Oaxaca: Picacho-San Gerónimo, Purpus 6810, oct 1913 (holotipo: UC!; isotipos: GH, NY!, US! ). Hierbas perennes, erectas, $2.0 \mathrm{~m}$ de altura. Tallos huecos, estriados, uncinados o glabrescentes. Hojas 3-folioladas; estípulas persistentes, libres, 6.5-8.5 mm largo, 1.0-1.8 $\mathrm{mm}$ ancho, lanceoladas a ovadas, largo atenuadas, adaxialmente hírtulas, abaxialmente glabras, ciliadas; pecíolos estriados, 1.0-5.5 cm largo, uncinados; estipelas 1.5-5.0 mm largo, ca. $0.6 \mathrm{~mm}$ ancho, largamente atenuadas en el ápice, adaxialmente puberulentas y uncinadas, 
abaxialmente glabras, ciliadas; peciólulos 2.0-3.0 mm largo, tomentosos; folíolo terminal $3.5-10.0 \mathrm{~cm}$ largo, 1.5$6.5 \mathrm{~cm}$ ancho, los laterales $2.7-8.0 \mathrm{~cm}$ largo, $1.0-4.5 \mathrm{~cm}$ ancho, trulados, ovados a lanceolados, base cuneada, ápice agudo, haz hirsuto, envés hirsútulo. Inflorescencias axilares y terminales, en panículas, $10.0-28.0 \mathrm{~cm}$ largo; eje de la inflorescencia uncinado; brácteas primarias persistentes, $1.7 \mathrm{~mm}$ largo, $0.4 \mathrm{~mm}$ ancho, subuladas, adaxialmente puberulentas y uncinadas, abaxialmente glabrescentes, ciliadas; brácteas secundarias persistentes $5.0 \mathrm{~mm}$ largo, $0.7 \mathrm{~mm}$ ancho, adaxialmente glabrescentes, abaxialmente glabras, ciliadas; pedicelos cortos 5.0-8.0 mm largo, uncinados, puberulentos y glandulosos. Flores blancas, 4-6 por nudo; cáliz $2.0 \mathrm{~mm}$ largo, adaxialmente hirsuto, abaxialmente glabrescente, lóbulo superior 2-dividido, $1.7 \mathrm{~mm}$ largo, lóbulo inferior con dientes laterales 1.5-1.7 mm largo, diente central $1.7 \mathrm{~mm}$ largo; corola ca. $3.5 \mathrm{~mm}$ largo, estandarte $2.3 \mathrm{~mm}$ largo, $1.6 \mathrm{~mm}$ ancho, oblongo, alas 2.0 $\mathrm{mm}$ largo, $0.9 \mathrm{~mm}$ ancho, oblanceoladas, quilla 2.2-2.4 mm largo; ovario sésil, glabrescente, 3 óvulos. Lomentos aplanados, sésiles; artículos 3-4, 8.0-9.0 mm largo, 5.0-6.0 $\mathrm{mm}$ ancho, generalmente 3 artículos, espiralados, puberulentos y uncinados, ciliados (dos no se desarrollan), el terminal, fértil, oblongo con margen crenado y ciliado, glabrescente, indehiscente; semillas 1.0-2.0 mm largo, 1.4$1.6 \mathrm{~mm}$ ancho, oblongas.

Distribución y hábitat. En Campeche, Yucatán y escasa en Quintana Roo (Fig. 2). En otras entidades de México (Baja California Sur, Chiapas, Guerrero, Jalisco, Michoacán, Morelos, Nayarit, Oaxaca, Puebla, Sinaloa, Tamaulipas y Veracruz), Centroamérica, Antillas y Sudamérica. Se establece en dunas costeras, selva baja caducifolia, selva media subcaducifolia, selva media subperennifolia y vegetación secundaria. Florece de octubre a marzo y fructifica de agosto a abril.

Comentarios taxonómicos. Desmodium glabrum crece comúnmente en áreas perturbadas muy cercana a D. procumbens, en especial en orillas de caminos, y es fácilmente reconocible de las demás especies de la península por sus panículas que desarrollan frutos glabrescentes de un sólo artículo fértil.

Usos y nombres comunes. Existen algunos registros sobre su utilización como planta forrajera. Se le conoce localmente con los nombres de: kintal, kiintaj, o xbul kaax (maya).

Material examinado. Campeche: C. Chan 4285 (MEXU); D. Álvarez 7335, 7769 (MEXU); E. Cabrera 12805, 15214 (MEXU); E. Martínez 29528 (CICY, MEXU), 29979 (MEXU); I. Calzada 6698 (CICY, MEXU); P. Zamora 5898 (CICY, MEXU); R. Durán 1421 (CICY, MEXU, XAL); Quintana Roo: O. Téllez 1379 (MEXU); Yucatán: C. Vargas 197 (CICY, MEXU); E. Cabrera 9403, 13271 (MEXU); E. Estrada 116 (CICY, ENCB, MEXU); E.
Reyes 589, 879 (MEXU); F. Ku Yam 526, 894 (MEXU); G. Davidse 29442 (MEXU); I. Calzada 6504 (CICY, MEXU); J.S. Flores 9598, 10335 (CICY, MEXU); M.A. Magaña 511 (CICY, ENCB, MEXU); M. Narváez 167 (CICY, MEXU); M.A. Pérez 527, 637, 727, 789 (MEXU); O. Téllez 6444 (CICY, MEXU); P. Yam 21 (CICY, MEXU); $R$. Conway 508-79 (MEXU); R. Duno 1836 (CICY, MEXU); S. Darwin 2093 (MEXU).

Desmodium incanum DC., Prodr. 2:332. 1825. Hedysarum incanum Sw., Prodr. 107. 1788. Meibomia incana (DC.) Vail, Bull. Torrey Bot. Club 19:118. 1892. Desmodium canum Schinz et Tell., Mém. Soc. Sci. Nat. Neuchâtel 5:371. 1914. Meibomia cana S.F. Blake, Bot. Gaz. 78:276. 1924. Tipo: Antillas, Plumier, Pl. Amer. t. 149, fig. 1. 1757 (problamente en PY).

Hierbas perennes, erectas o procumbentes, $1.5 \mathrm{~m}$ de altura. Tallos estoloníferos, estriados, puberulentos y uncinados. Hojas 3-folioladas, en ocasiones las basales 1-folioladas; estípulas persistentes, unidas en el lado opuesto del tallo, 5.0-7.0 mm largo, 1.8-2.0 mm ancho, ovadas a lanceoladas, adaxialmente uncinadas y escasamente hirsutas, abaxialmente glabras, ciliadas; pecíolos sulcados, 1.0-3.0 cm largo, uncinados; estipelas 2.0-3.0 mm largo, 0.2-0.4 $\mathrm{mm}$ ancho, subuladas, adaxialmente puberulentas y uncinadas, abaxialmente glabras, ciliadas; peciólulos 2.0-2.5 $\mathrm{mm}$ largo, híspidos; folíolo terminal $3.5-9.3 \mathrm{~cm}$ largo, $2-4.5 \mathrm{~cm}$ ancho, los laterales $2-6.5 \mathrm{~cm}$ largo, $1.5-2.5 \mathrm{~cm}$ ancho, ovados, ovado-lanceolados a elípticos, base corto atenuada, ápice obtuso, haz escasamente uncinado o glabrescente, envés densamente hírtulo. Inflorescencias axilares y terminales, $9.0-24.0 \mathrm{~cm}$ largo; eje de la inflorescencia uncinado; brácteas primarias deciduas, $2.0 \mathrm{~mm}$ largo, $0.3 \mathrm{~mm}$ ancho, subuladas, adaxialmente uncinadas, abaxialmente glabras, ciliadas; brácteas secundarias deciduas, $1.0 \mathrm{~mm}$ largo, $0.7 \mathrm{~mm}$ ancho, subuladas, glabras en ambas superficies, ciliadas; pedicelos de ca. $5.0 \mathrm{~mm}$ de largo, uncinados. Flores de color lila, 2 por nudo; cáliz $2.5 \mathrm{~mm}$ largo, adaxialmente hírtulo, abaxialmente glabro, lóbulo superior 2-dividido, $1.8 \mathrm{~mm}$ largo, lóbulo inferior con dientes laterales 1.6-1.8 mm largo, diente central 1.6 $\mathrm{mm}$ largo; corola 6.0-7.0 mm largo, estandarte $5.0 \mathrm{~mm}$ largo, $3.2 \mathrm{~mm}$ ancho, obovado, alas $5.0 \mathrm{~mm}$ largo, 1.2-1.5 $\mathrm{mm}$ ancho, oblongas, quilla $5.0 \mathrm{~mm}$ largo; ovario sésil, con escasos tricomas uncinados, 8 óvulos. Lomentos aplanados, sésiles; artículos 6-8, 3.0-4.0 mm largo, 1.5$2.5 \mathrm{~mm}$ ancho, oblongos, margen superior ligeramente sinuado a recto, margen inferior profundamente crenado, densamente uncinados y puberulentos, indehiscentes; semillas $1.5 \mathrm{~mm}$ largo, $1.0 \mathrm{~mm}$ ancho, elípticas.

Distribución y hábitat. Desmodium incanum está ampliamente distribuida en toda la península (Fig. 1). Desde el S 
de Estados Unidos, en otras entidades de México (Chiapas, Guerrero, Hidalgo, Jalisco, Nayarit, Nuevo León, Oaxaca, Puebla, San Luis Potosí, Tabasco, Tamaulipas y Veracruz), Centroamérica, Antillas, Sudamérica y ha sido introducida al Viejo Mundo. Crece en dunas costeras, manglar, sabana, selva baja inundable, selva media subcaducifolia, selva media subperennifolia, selva alta perennifolia y vegetación secundaria. Florece y fructifica todo el año.

Comentarios taxonómicos. Desmodium incanum se caracteriza por su hábito sufruticoso, tallo estolonífero, hojas lanceoladas a ovadas, envés estrigoso y haz pálido, su lomento generalmente desarrolla 8 artículos.

Usos y nombres comunes. Se utiliza como forrajera y medicinal. Se le conoce con los nombres de: b uul-bech, kabah, k’axá yuuh, tza-yun-tzay, xcaxayuc, x-komhá (maya) y pega pega (español).

Material examinado. Campeche: B. ECOMAT 16 (CICY); C. Chan 741, 3908 (CICY); C. Gutiérrez 4336, 4969 (MEXU), 7161 (MEXU, UCAM); C. Martín 514 (MEXU); C. Pavón 177 (CICY, MEXU); D. Álvarez 15, 414, 517, 3588, 4488, 9678 (MEXU); D. Mondragón 34 (CICY, MEXU); E. Cabrera 1971, 4476, 11889, (MEXU), 12588 (IEB, MEXU); E. Hernández 232 (MEXU); E. Lira 151, 186, 253, 271, 437 (MEXU); E. Madrid 4, 30, 139, 581, 792, 810, 1031 (MEXU); E. Martínez 2702, 27913, 28072, 28697, 28786- $A$ (MEXU), 29454 (CICY, MEXU), 29832, 31508a, 31835, 35044, 35062, $35264 a$ (MEXU); G. Bacab 86 (CICY, MEXU); J. Calónico 21614, 22695, 23866 (MEXU); J. Palmillas 869 (MEXU); J.S. Flores 10295 (CICY, MEXU), 10316 (CICY); M. Sousa 12230 (MEXU); O. Téllez 6313 (ENCB, MEXU); P. Alvaro 179, 437 (MEXU); P. Zamora 4660, 5497 (CICY); Quintana Roo: A. Pérez 1590 (ENCB, MEXU), 1599 (MEXU); $C$. Chan 4335 (CICY); D. Álvarez 8073, 8385, 9430, 10379, 10475, 11095 (MEXU); E. Cabrera 252 (MEXU), 436 (ENCB, MEXU), 654, 881, 963, 1225, 1528, 1602, 6296, 8278, 9878, 11082, 11447, 13015, 16088, 16391 (MEXU); E. Ucan 1305 (CICY, MEXU, XAL), 3248 (CICY); $G$. Carnevali 4868 (CICY, MEXU); J. Calónico 22359, 22292, 22547 (MEXU); J.J. Ortiz 472 (MEXU, XAL); J.L. Tapia 1013 (CICY, MEXU); J.S. Flores 401, 9976, 10163 (CICY), 10511 (MEXU); M. Sousa 10918, 11220 (MEXU); O. Téllez 1727, 1820, 2086, 2168 (MEXU), 2656 (CICY, MEXU), 2749 (MEXU); R. Durán 801 (MEXU); R. Sánchez 147, 467, 483, 517, 546, 624, 639, $669,1120,1148,1162,1180$ (MEXU); R. Villanueva 690 (MEXU); S. Escalante 14 (MEXU); Yucatán: A. Maurilio 23 (MEXU); C.D. Johnson 1752-80 (MEXU); E. Reyes 439, 701 (MEXU); F. Ku Yam 778, 873 (MEXU); G. Remmers 14 (CICY); J.A. Aguilar 177 (CICY, MEXU), 274 (MEXU); M.A. Pérez 582, 853 (MEXU); M. Narváez 379 (CICY, XAL); O.G. Enríquez 218 (MEXU); R. Grether
1335 (MEXU), 1338 (ENCB); S. Escalante 3565 (CICY); W.C. Steere 1460 (MEXU).

Desmodium procumbens (Mill.) Hitchc., Ann. Rep. Missouri Bot. Gard. 4:76. 1893. Hedysarum procumbens Mill., Gard. Dict., ed. 8. Hedysarum no. 10. 1768. Meibomia procumbens (Mill.) Britton, Sci. Surv. Porto Rico and Virgen Islands 5:404. 1924. Tipo: Jamaica: Houstoun s.n., 1730 (holotipo: BM!). Desmodium sylvaticum Benth., Pl. Hartw. 116. 1843. Meibomia procumbens (Mill.) Schindl. var. sylvatica (Benth.) Schindl., Repert. Spec. Nov. Regni Veg. 20:151. 1924. Tipo: Ecuador. Guayas, Guayaquil, Hartweg 650, 1839 (holotipo: K; isotipos: GH, NY).

Hierbas anuales, procumbentes, $2.0 \mathrm{~m}$ de largo. Tallos ramificados, estriados, uncinados. Hojas 3-folioladas, ocasionalmente 1-foliolada; estípulas persistentes, libres, 3.0-7.0 mm de largo, $0.5 \mathrm{~mm}$ ancho, subuladas, glabras en ambas superficies, ciliadas; pecíolos sulcados, $1.0-4.5 \mathrm{~cm}$ largo, escasamente uncinados o glabrescentes; estipelas 0.8-3.5 mm largo, ca. $0.2 \mathrm{~mm}$ ancho, lineares, glabrescentes en ambas superficies, ciliadas; peciólulos 1.0-2.0 $\mathrm{mm}$ largo, hirsutos; folíolo terminal $2.0-5.5 \mathrm{~cm}$ largo, 0.8-2.2 cm ancho, los laterales 1.5-4.5 cm largo, 0.9-2.2 $\mathrm{cm}$ ancho, ovados, lanceolados o elípticos, base atenuada, ápice agudo, glabrescentes en ambas superficies. Inflorescencias axilares y terminales, 6.0-22 cm largo; eje de la inflorescencia puberulento y escasamente uncinado; brácteas primarias persistentes, $2.2 \mathrm{~mm}$ largo, $0.3 \mathrm{~mm}$ ancho, subuladas, glabrescentes en ambas superficies, ciliadas; brácteas secundarias persistentes, $1.0 \mathrm{~mm}$ largo, 0.2 $\mathrm{mm}$ ancho, lineares, puberulentas en ambas superficies, ciliadas; pedicelos $1.0 \mathrm{~cm}$ de largo, uncinados y pilosos. Flores blancas, 2 (-5) o más por nudo; cáliz $1.3 \mathrm{~mm}$ largo, adaxialmente estrigoso, abaxialmente glabro, lóbulo superior 2-dividido, $1.0 \mathrm{~mm}$ largo, lóbulo inferior con dientes laterales $1.0 \mathrm{~mm}$ largo, diente central $1.0 \mathrm{~mm}$ largo; corola $3.0 \mathrm{~mm}$ largo, estandarte 2.3-2.5 mm largo, 2.0-2.1 mm ancho, ovado, alas $2.0 \mathrm{~mm}$ largo, $0.8 \mathrm{~mm}$ ancho, oblongas, quilla $2.7 \mathrm{~mm}$ largo; ovario sésil, pubescente, 6 óvulos. Lomentos espiralados, sésiles; artículos 3-5, 4.0-6.0 mm largo, 2.5-3.5 $\mathrm{mm}$ ancho, rómbicos, margen involuto $\mathrm{y}$ revoluto, uncinados, indehiscentes; semillas $1.5 \mathrm{~mm}$ largo, ca. $1.0 \mathrm{~mm}$ ancho, rómbicas.

Distribución y hábitat. En la zona de estudio, está escasamente representada en Campeche y Quintana Roo y más ampliamente distribuida en Yucatán (Fig. 3). Desde el SO de Estados Unidos, en otras entidades de México (Baja California Sur, Chiapas, Chihuahua, Colima, Distrito Federal, Guanajuato, Guerrero, Hidalgo, Jalisco, México, Michoacán, Morelos, Nayarit, Oaxaca, Puebla, Querétaro, Sinaloa, Sonora, Veracruz y Zacatecas), Centroamérica, Antillas, Sudamérica e introducida en regiones tropicales 
de Asia y África. Habita en la selva baja caducifolia, selva mediana subcaducifolia y vegetación secundaria. Florece entre agosto y diciembre y fructifica entre septiembre y diciembre.

Comentarios taxonómicos. En ocasiones, Desmodium procumbens puede confundirse con $D$. tortuosum en etapas jóvenes de desarrollo, pero sus estípulas subuladas, el desarrollo de hasta 5 flores por nudo y la persistencia de sus brácteas primarias y secundarias, la distinguen de esta última.

Nombre común. Localmente se le conoce con el nombre común de k’iintaj (maya).

Material examinado. Campeche: C. Gutiérrez 8014 (MEXU); E. Cabrera 9492 (MEXU); E. Martínez 28912-A (MEXU); I. Calzada 6708 (MEXU, XAL); Quintana Roo: E. Cabrera 506 (MEXU); Yucatán: E. Cabrera 9440, 9589, 9680, 9742 (MEXU); E. Estrada 352 (CICY, MEXU); E. Reyes 91, 293, 294, 481, 599, 790 (MEXU); E. Ucan 166 (CICY, MEXU); F. May 1302 (CICY, MEXU); F. Tun 362 (ENCB, MEXU); G.F. Gaumer 1118 (MO); L. M. Arias 608 (CHAPA, MEXU); M.A. Pérez 498, 508, 511, 719, 782, 788, 887 (MEXU); V. Rico-Gray 774 (MEXU).

Desmodium scorpiurus (Sw.) Desv., J. Bot. Agric. 1:122. 1813.

Hedysarum scorpiurus Sw., Prod. 107. 1788. Meibomia scorpiurus (Sw.) Kuntze, Revis. Gen. Pl. 1: 198. 1891. Tipo: Jamaica: Swartz s.n., s.f. (holotipo: S).

Nissoloides cilindrica M.E. Jones, Contr. W. Bot. 18:135. 1933. Tipo: México: Guadalajara, M.E. Jones 27223, 27 Nov 1930 (isotipos: GH, US!).

Hierbas perennes, postradas, $1.0 \mathrm{~m}$ de largo. Tallos estriados, puberulentos o escasamente uncinados. Hojas 3-folioladas; estípulas persistentes, connadas, $2.0-2.5 \mathrm{~mm}$ largo, 1.3-1.6 mm ancho, ovadas a ovado lanceoladas, auriculadas en su base, glabras en ambas superficies, ciliadas; pecíolos sulcados, 1.0-3.0 cm largo, pilosos; estipelas 1.0-1.7 mm largo, $0.4 \mathrm{~mm}$ ancho, lineares, adaxialmente uncinadas, abaxialmente glabras, ciliadas; peciólulos 1.0$1.5 \mathrm{~mm}$ largo, hirsutos; folíolo terminal $1.7-2.5 \mathrm{~cm}$ largo, 0.4-0.8 cm ancho, los laterales $1.3-2.0 \mathrm{~cm}$ largo, $0.3-0.7 \mathrm{~cm}$ ancho, elípticos a ovados, base atenuada, ápice agudo, haz adpreso-piloso, envés glabrescente. Inflorescencias axilares y terminales, 3.0-15.5 cm largo; eje de la inflorescencia puberulento y uncinado; brácteas primarias persistentes, $2.0 \mathrm{~mm}$ largo, $0.4 \mathrm{~mm}$ ancho, subuladas, glabrescentes en ambas superficies, ciliadas; brácteas secundarias persistentes, $1.1 \mathrm{~mm}$ largo, $0.2 \mathrm{~mm}$ ancho, subuladas, adaxialmente puberulentas y uncinadas, abaxialmente glabras, ciliadas; pedicelos $1.0 \mathrm{~cm}$ de largo, puberulentos y uncinados. Flores de color lila, 2 por nudo; cáliz $2.7 \mathrm{~mm}$ largo, adaxialmente hirsútulo, abaxialmente glabrescente, lóbulo superior 2-dividido, $2.0 \mathrm{~mm}$ largo, lóbulo inferior con dientes laterales 1.7-1.8 mm largo, diente central 1.7 $\mathrm{mm}$ largo; corola $4.0 \mathrm{~mm}$ largo, estandarte $5.0 \mathrm{~mm}$ largo, $5.5 \mathrm{~mm}$ ancho, subrotundo, alas $3.8-4.1 \mathrm{~mm}$ largo, $1.8 \mathrm{~mm}$ ancho, oblongas, quilla $5.0 \mathrm{~mm}$ largo; ovario sésil, glabrescente, 8 óvulos. Lomentos aplanados, lineares, sésiles; artículos 6-8, 4.5-5.1 mm largo, 1.5-1.7 mm ancho, oblongos, margen ligeramente sinuados, escasamente uncinados o glabrescentes, indehiscentes; semillas 2.0-3.2 mm largo, ca. $1.2 \mathrm{~mm}$ ancho, oblongas.

Distribución y hábitat. En la región, Desmodium scorpiurus es poco frecuente. Se le conoce únicamente de Campeche (Fig. 2), SE de Estados Unidos y más ampliamente distribuida en otras regiones de México (Baja California Sur, Chiapas, Colima, Durango, Guerrero, Hidalgo, Jalisco, México, Michoacán, Morelos, Nayarit, Oaxaca, Puebla, San Luis Potosí, Sinaloa, Sonora, Tabasco, Tamaulipas y Veracruz), Centroamérica, Antillas, N de Sudamérica e introducida a los trópicos de Asia y África. Crece en dunas costeras, selva baja caducifolia, selva media subcaducifolia y vegetación secundaria. Florece entre junio y marzo y fructifica entre noviembre y marzo.

Comentarios taxonómicos. Desmodium scorpiurus es la única especie del género que desarrolla lomentos lineares. Material examinado. Campeche: E. Cabrera 2325 (ENCB, MEXU), 10891, 14836 (MEXU).

Desmodium tortuosum (Sw.) DC., Prodr. 2:332. 1825. Hedysarum tortuosum (Sw.) DC., Prodr. 107. 1788. Meibomia tortuosa (Sw.) Kuntze, Revis. Gen. Pl. 1: 198. 1891. Tipo: Jamaica: sin localidad, Swartz s.n., s.f. (holotipo: BM!). Hedysarum purpureum Mill. Gard. Dict. ed. 8. Hedysarum no. 6. 1768. Desmodium purpureum (Mill.) Fawc. et Rendle, Fl. Jamaica 4: 36. 1920, non $D$. purpureum Hook. et Arn. 1832. Tipo: México. Veracruz, Houstoun s.n., 1730. (holotipo: BM!). Desmodium stipulaceum DC., Prodr. 2:330. 1825. Meibomia stipulacea (DC.) Kuntze, Revis. Gen. Pl. 1:198. 1891. "Hedysarum stipulaceum fl. mex. icon. ined.".

Tipo: México. Sin localidad, Sessé y Mociño s.n., s.f. (lectotipo: F!, designado por McVaugh, 2000).

Hierbas perennes, erectas, $2.0 \mathrm{~m}$ de altura. Tallos estriados, híspidos y uncinados. Hojas 3-folioladas; estípulas persistentes, amplexicaules, $0.8-1.5 \mathrm{~cm}$ largo, 0.3-0.5 cm ancho, ovadas a ovado-lanceoladas, glabras en ambas superficies, ciliadas; pecíolos estriados, $2.0-5.5 \mathrm{~cm}$ largo, hirsutos y uncinados; estipelas 4.5-8.5 mm largo, $0.5 \mathrm{~mm}$ ancho, subuladas, glabrescentes en ambas superficies, ciliadas; peciólulos ca. $4.0 \mathrm{~mm}$ largo, híspidos; folíolo terminal 4.0-11.0 cm largo, 2.0-4.0 cm ancho; los laterales 3.0-7.0 cm largo, 1.2-3.8 cm ancho, ovados, ovado-lanceolados a elípticos, base redondeada, ápice agudo a redondeado, 
glabrescentes en ambas superficies. Inflorescencias axilares y terminales, en panículas 9.0-33.5 cm largo; eje de la inflorescencia uncinado y glanduloso; brácteas primarias deciduas, 4.8-5.2 $\mathrm{mm}$ largo, $0.7 \mathrm{~mm}$ ancho, ovado acuminadas, glabras en ambas superficies, ciliadas; brácteas secundarias deciduas, $2.2 \mathrm{~mm}$ largo, $0.3 \mathrm{~mm}$ ancho, subuladas, glabras en ambas superficies, ciliadas; pedicelos 1.7 $\mathrm{cm}$ de largo, uncinados y glandulosos. Flores de color lila, 2 por nudo; cáliz 3.0-3.5 mm largo, adaxialmente hirsuto, abaxialmente glabro, lóbulo superior 2-dividido, $2.3 \mathrm{~mm}$ largo, lóbulo inferior con dientes laterales 1.8-2.0 mm largo, diente central $2.1 \mathrm{~mm}$ largo; corola $5.5 \mathrm{~mm}$ largo, estandarte ca. $3.8 \mathrm{~mm}$ largo, $1.8 \mathrm{~mm}$ ancho, obovado, alas $3.8 \mathrm{~mm}$ largo, $1.5 \mathrm{~mm}$ ancho, oblongas, quilla $5.0 \mathrm{~mm}$ largo; ovario cortamente estipitado, puberulento, 6 óvulos. Lomentos aplanados, sésiles; artículos 2-7, 4.3-6.0 $\mathrm{mm}$ largo, 3.0-4.0 $\mathrm{mm}$ ancho, elípticos, margen crenado, cuando inmaduros involutos y revolutos, esparcidamente uncinados, indehiscentes; semillas 2.6-2.8 mm largo, 1.5$2.0 \mathrm{~mm}$ ancho, elípticas.

Distribución y hábitat. Ampliamente distribuida en toda la península de Yucatán (Fig. 4), SO de Estados Unidos, otras entidades de México (Chiapas, Chihuahua, Coahuila, Colima, Durango, Guerrero, Hidalgo, Jalisco, México, Michoacán, Morelos, Nayarit, Nuevo León, Oaxaca, Puebla, Querétaro, San Luis Potosí, Sinaloa, Sonora, Tabasco, Tamaulipas, Veracruz y Zacatecas), Centroamérica, Antillas, Sudamérica e introducida a los trópicos de Asia y África. Crece en dunas costeras, selva baja caducifolia, selva media subcaducifolia, selva media subperennifolia y vegetación secundaria. Florece y fructifica de junio a abril. Comentarios taxonómicos. Morfológicamente, Desmodium tortuosum es muy similar a $D$. distortum en su forma de crecimiento y sus estípulas amplexicaules; sin embargo D. tortuosum desarrolla tricomas uncinados mezclados con tricomas híspidos en tallos y pecíolos, lomentos sésiles, generalmente uncinados y puberulentos, que permiten diferenciarla.

Usos y nombres comunes. Es utilizada como planta forrajera. Localmente se conoce como bul kax, bu' ul lk' $a x$, buultsimin-buulxiu, kintah, kiintaj, xkintalxiw, xk' intaj, oxkutzcab, xpacumpak (maya).

Material examinado. Campeche: C. Chan 894, 931 (CICY, MEXU, XAL), 4721 (CICY, MEXU); C. Gutiérrez 5454 (MEXU); C.L. Lundell 960 (MEXU); Conway 533-79 (MEXU); C. Pavón 206, 227 (CICY, MEXU); D. Álvarez 2015 (MEXU); D. Álvarez 2051, 2357, 4468 (MEXU); E. Cabrera 2016, 2258, 12489, 14516, 14568, 15264 (MEXU); E. Lira 466, 616-A, 643 (MEXU); E. Martínez 3002, 29548, 29560-A, 29564, 29620, 29770 (MEXU); E. Ucan 1610 (CICY, MEXU, XAL), 1637 (CICY, XAL); G. Bacab 164 (CICY, MEXU); J. Caló-

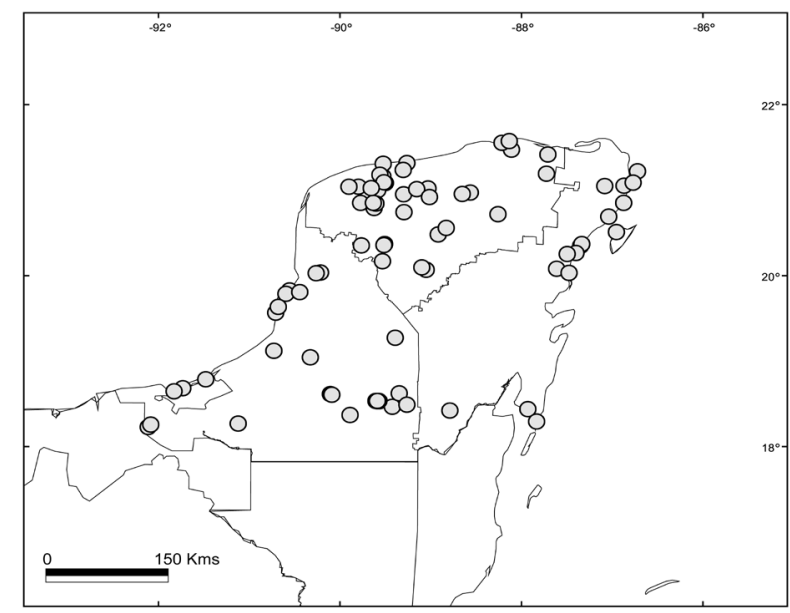

Figura 4. Distribución de Desmodium tortuosum (•).

nico 21716 (MEXU); J.S. Flores 10301 (CICY); O. Vela 721 (MEXU); P. Moreno 1137 (MEXU); P. Zamora 5907 (MEXU, UCAM); Quintana Roo: C. Chan 13 (MEXU); C.D. Johnson 426-78 (MEXU); E. Cabrera 210, 359 (CICY, MEXU), 470, 479, 558, 751, 921, 1462, 8295, 9761, 15464 (MEXU); E. Gutiérrez 172 (CICY, MEXU); E. Ucan 3626 (CICY); I. Calzada 7153 (CICY, XAL); M. Sousa 10874, 12444 (MEXU); P. Herrera 53 (MEXU); $R$. Durán 543 (MEXU); S. Torres 491 (CICY, MEXU); Yucatán: A. Puch 674, 1090 (CICY); C. Chan 6768 (CICY, MEXU); E. Cabrera 9455, 9997, 10074 (MEXU), 10443 (CICY, MEXU), 10656, 13272 (MEXU); E. Reyes 100, 109, 294a, 537, 601, 768, 791, 842 (MEXU); E. Ucan 176, 1829, 3577 (CICY, MEXU); G.F. Gaumer 1021 (MEXU); I. Calzada 6637 (CICY); J. Leal 85 (CICY, MEXU); J.S. Flores 9013 (CICY, MEXU, XAL); M.A. Pérez 164, 512, 631, 733, 886 (MEXU); M. Narváez 765 (CICY, MEXU, XAL); O.G. Enríquez 646, 816 (MEXU); O.L. Sanabria 55-258 (CICY); P. Simá 326, 2336 (CICY, MEXU); P. Yam 75 (CICY, MEXU); R. Duno 1837 (CICY, MEXU); R. Rivera 141, 229 (CICY, MEXU); S. Darwin 2092 (CICY, MEXU).

Desmodium triflorum (L.) DC., Prodr. 2:334. 1825. Hedysarum triflorum L., Sp. Pl. 749. 1753. Meibomia triflora (L.) Kuntze, Revis. Gen. P1. 1:97. 1891. Tipo: India. Ceilán, Herb. Hermann 1:21, No. 297 (lectotipo: BM, designado por Pedley, 1997).

Hierbas perennes, postradas, 20-60 cm largo. Tallos ramificados, estriados, densamente pilosos. Hojas 3-folioladas, ocasionalmente 1-folioladas; estípulas persistentes, libres, 2.0-2.6 $\mathrm{mm}$ de largo, $1.0 \mathrm{~mm}$ ancho, lanceoladas, glabras en ambas superficies, ciliadas; pecíolos sulcados, 3.0-5.0 mm largo, pilosos; estipelas ca. $1.0 \mathrm{~mm}$ largo, $0.1 \mathrm{~mm}$ ancho, lineares, ciliadas; peciólulos ca. $1.0 \mathrm{~mm}$ largo, densamente 
pilosos; folíolo terminal $6.5-10.0 \mathrm{~mm}$ largo, $6.2-10.0 \mathrm{~mm}$ ancho, los laterales 5.8-9.0 $\mathrm{mm}$ de largo, $4.5-9.0 \mathrm{~mm}$ de ancho, obovados, base cuneada, ápice retuso, haz glabro, envés puberulento a lo largo de las vena principal y venas laterales. Inflorescencias axilares, en fascículos, 1.5-2.5 cm largo; eje de la inflorescencia piloso; brácteas primarias persistentes, $2.5-3.0 \mathrm{~mm}$ largo, 1.2-1.3 mm ancho, ovadas, glabrescentes en ambas superficies, ciliadas; brácteas secundarias deciduas (no vistas); pedicelos $8.0 \mathrm{~mm}$ de largo, densamente hirsutos. Flores rosa o lila, 2-4 por fascículo; cáliz $3.5 \mathrm{~mm}$ largo, adaxialmente hirsuto, abaxialmente glabro, lóbulo superior 2-dividido, $2.2 \mathrm{~mm}$ largo, lóbulo inferior con dientes laterales $2.0 \mathrm{~mm}$ largo, diente central $1.6 \mathrm{~mm}$ largo; corola 4.0-6.0 mm largo, estandarte $3.2 \mathrm{~mm}$ largo, $2.3 \mathrm{~mm}$ ancho, oblado, alas $2.8-3.0 \mathrm{~mm}$ largo, 1.0-1.3 mm ancho, espatuladas, quilla $3.0 \mathrm{~mm}$ largo; ovario sésil, puberulento, 5 óvulos. Lomentos aplanados, falcados, sésiles, artículos 3-5, 3.2-3.5 mm largo, 2.5-3 mm ancho, cuadrados, margen superior ligeramente sinuado, margen inferior crenado, glabrescentes, indehiscentes; semillas 2.0-2.1 mm largo, 1.5-1.6 mm ancho, orbiculares. Distribución y hábitat. Se conocen pocas recolecciones para la porción mexicana de la península de Yucatán (Fig. 1). Introducida en el SE de Estados Unidos, en otras entidades de México (Chiapas, Colima, Guerrero, Jalisco, Nayarit, Oaxaca, Puebla, San Luis Potosí, Sinaloa, Tabasco, Tamaulipas y Veracruz), Centroamérica, Antillas, Brasil, África, Asia y Australia. Habita en dunas costeras. Florece en noviembre.

Comentarios taxonómicos. Desmodium triflorum es la única especie del género que desarrolla hábito postrado, con los tallos muy ramificados, hojas pequeñas, obovadas o anchamente obovadas de ápice retuso e inflorescencias fasciculadas con 2-4 flores.

Material examinado. Campeche: E. Cabrera 14835 (CIQRO, IEB, MEXU).

Especies excluidas

Desmodium adscendens (Sw.) DC. Citada en algunas listas florísticas de la península (Standley 1930; Sousa y Cabrera 1983; Sosa et al. 1985; Durán et al. 2000; Arellano-Rodríguez et al. 2003), donde se hace referencia al ejemplar de G. F. Gaumer s. n., recolectado en 1858. Aunque Durán et al. (2000) la citan para el Herbario Nacional (MEXU), sólo se localizaron 2 ejemplares; uno en el Field Museum of Naturall History (F) y el otro en el Herbarium, Royal Botanic Garden, Kew (K), ambos corresponden a Desmodium tortuosum.

Desmodium helleri Peyr. Citada en el listado florístico de Campeche (Gutiérrez-Báez, 2003); sin embargo, la recolección de C. Gutiérrez 5046, corresponde a Desmodium incanum.

Desmodium purpureum (Mill.) Fawc. et Rendle
Se menciona en trabajos como el de Standley (1930); Sosa et al. (1985); Gutiérrez-Báez (2003) y Arellano-Rodríguez et al. (2003). Standley (1930) mencionan la recolección de G. F. Gaumer 1118, que corresponde a Desmodium procumbens. El ejemplar E. Ucan 1872 citado en Etnoflora Yucatanense (Sosa et al. 1985) corresponde a Desmodium distortum y el ejemplar C. Lundell 960, citado por Gutiérrez-Báez (2003) corresponde a Desmodium tortuosum. Lamentablemente, Arellano-Rodríguez et al. (2003) no incluye ejemplares de referencia en su listado.

Desmodium procumbens var. transversum (B.L. Rob. et Greenm.) B.G. Schub.

El ejemplar de E. Ucan 1639, citado en el listado florístico de Campeche por Gutiérrez-Báez (2003), corresponde a la variedad típica de $D$. procumbens.

\section{Agradecimientos}

A los curadores de los herbarios consultados. A la Dra. Lourdes Rico y al M. en C. Rafael Torres Colín, por la revisión del manuscrito. Al Dr. Alfonso Delgado Salinas, por sus valiosos comentarios y al Biól. Alfredo Wong León, por su apoyo técnico.

\section{Literatura citada}

Arellano-Rodríguez, J. A., J. S. Flores-Guido, J. Tun-Garrido y M. M. Cruz-Bojórquez. 2003. Nomenclatura, forma de vida, uso, manejo y distribución de las especies vegetales de la península de Yucatán. In Etnoflora Yucatanense 20. Universidad Autónoma de Yucatán, Mérida. 815 p.

Barrera, A. 1962. La Península de Yucatán como provincia biótica. Revista de la Sociedad Mexicana de Historia Natural 23:71-105.

Breedlove, D. E. 1986. Listados Florísticos de México IV. Flora de Chiapas. Instituto de Biología, Universidad Nacional Autónoma de México, México, D. F. 246 p.

Cowan, C. P. 1983. Listados Florísticos de México I. Flora de Tabasco. Instituto de Biología, Universidad Nacional Autónoma de México, México, D. F. 123 p.

Durán, R., G. Campos, J. C. Trejo, P. Simá, F. May-Pat y M. JuanQui. 2000. Listado Florístico de la península de Yucatán. Centro de Investigación Científica de Yucatán, Mérida. 259 p.

Flores, J. S. 1992. Vegetación de las islas de la península de Yucatán. Florística y etnobotánica. In Etnoflora Yucatanense 4. Universidad Autónoma de Yucatán, Mérida. 71 p.

Gutiérrez-Báez, C. 2003. Listado florístico actualizado del estado de Campeche, México. Universidad Autónoma de Campeche, Campeche. México. 95 p.

Hemsley, W. B. 1887. Cozumel Island. Enumeration of a small collection of plants made in 1885 by F. Gaumer. Biologia- 
Centrali Americana, Botany 4:96-100.

Ibarra, G. y S. Sinaca. 1987. Listados Florísticos de México VII. Estación de Biología Tropical Los Tuxtlas, Veracruz. Instituto de Biología, Universidad Nacional Autónoma de México, México, D. F. 51 p.

INEGI (Instituto Nacional de Estadística, Geografía e Informática). 2000. XII Censo General de Población y Vivienda 2000. INEGI, Aguascalientes, Aguascalientes.104 p.

McVaugh, R. 1987. Leguminosae. In Flora Novo-Galiciana, W. R. Anderson (ed.). The University of Michigan Press, Ann Arbor. p. 448-496.

Morrone, J. J. 2005. Hacia una síntesis biogeográfica de México. Revista Mexicana de Biodiversidad 76:207-252.

Ohashi, H. 2005. Desmodieae. In Legumes of the world, G. Lewis, B. Schrire, B. Mackinder y M. Lock (eds.). Royal Botanic Gardens, Kew, Richmond. p. 433-446.

Rzedowski, J. 1978. La vegetación de México. Limusa, México, D. F. 432 p.

Sosa, V., J. S. Flores, V. Rico-Gray, R. Lira y J. J. Ortiz. 1985. Lista florística y sinonimia maya. In Etnoflora Yucatanense 1. Instituto Nacional de Investigaciones sobre Recursos Bióticos, Xalapa, Veracruz. 225 p.
Sousa, M. y E. F. Cabrera. 1983. Listados Florísticos de México. II. Flora de Quintana Roo. Instituto de Biología, Universidad Nacional Autónoma de México. México, D. F. 100 p.

Standley, P. C. 1930. Flora of Yucatan. Field Museum of Natural History. Botanical series 3:157-492.

Standley, P. C. y J. A. Steyermark. 1946. Flora of Guatemala. Fieldiana: Botany 24:232-233.

Téllez, O. y E. F. Cabrera. 1987. Listados Florísticos de México. VI. Flórula de la isla de Cozumel, Q. R., Instituto de Biología, Universidad Nacional Autónoma de México, México, D. F. $34 \mathrm{p}$.

Torres, C. R., L. Torres-Colín, P. Dávila y J. L. Villaseñor 1997. Listados Florísticos de México. XVI. Flora del distrito de Tehuantepec, Oaxaca. Instituto de Biología, Universidad Nacional Autónoma de México, México, D. F. 68 p.

Torres-Colín, L. Desmodium. Vegetación terrestre, Cap. 4. L. A. Pérez, M. Sousa, A. Hanan, F. Chiang y P. Tenorio. 2005. In Biodiversidad del estado de Tabasco, J. Bueno, F. Álvarez y S. Santiago (eds.). Instituto de Biología, UNAM/CONABIO, México, D. F. p. 65-110.

Torres-Colín, L. y A. Delgado-Salinas. 2008. Tribu Desmodieae. Desmodium. Flora del Valle de Tehuacán-Cuicatlán, Fascículo 59. 52 p. 